\title{
Adaptive control of a wearable exoskeleton for upper-extremity neurorehabilitation
}

\author{
Sivakumar Balasubramanian ${ }^{\mathrm{a}}$ and Jiping $\mathrm{He}^{\mathrm{b}, *}$ \\ ${ }^{a}$ Department of Bioengineering, Imperial College of Science, Technology and Medicine, London, UK \\ ${ }^{\mathrm{b}}$ School of Biological and Health Science Engineering, Arizona State University, Tempe, AZ, USA
}

\begin{abstract}
The paper describes the implementation and testing of two adaptive controllers developed for a wearable, underactuated upper extremity therapy robot - RUPERT (Robotic Upper Extremity Repetitive Trainer). The controllers developed in this study were used to implement two adaptive robotic therapy modes - the adaptive co-operative mode and the adaptive active-assist mode - that are based on two different approaches for providing robotic assistance for task practice. The adaptive active-assist mode completes therapy tasks when a subject is unable to do so voluntarily. This robotic therapy mode is a novel implementation of the idea of an active-assist therapy mode; it utilizes the measure of a subject's motor ability, along with their real-time movement kinematics to initiate robotic assistance at the appropriate time during a movement trial. The adaptive co-operative mode, on the other hand, is based on the idea of enabling task completion instead of completing the task for the subject. Both these therapy modes were designed to adapt to a stroke subject's motor ability, and thus encourage voluntary participation from the stroke subject. The two controllers were tested on three stroke subjects practicing robot-assisted reaching movements. The results from the testing demonstrates that an underactuated wearable exoskeleton, such as RUPERT, can be used for administering robot-assisted therapy, in a manner that encourages voluntary participation from the subject undergoing therapy.
\end{abstract}

Keywords: Stroke, robot-assisted neurorehabilitation, wearable exoskeleton, active-assist therapy, adaptive assistance

\section{Introduction}

Over the past 15 years there has been an increased interest in the use of robotic devices as tools for neurorehabilitation for the upper (and also the lower) extremity [1-4]. The purpose of these robotic devices is to provide sensorimotor stimulation to the subject undergoing robot-assisted therapy. This sensorimotor stimulation can range from the simple mechanical assistance for task completion [5-7] to the simulation of complex force fields to teach specific movement patterns [8].

In a previous article [9] we had described the design and development of RUPERT III (Fig. 1), a

\footnotetext{
*Corresponding author: Jiping He, Professor, School of Biological and Health Science Engineering, Arizona State University, Tempe, AZ, USA. E-mail: jiping.he@asu.edu.
}

wearable robotic exoskeleton developed by our group at the Arizona State University, and Kinetic Muscles Inc. (Tempe, Arizona, USA). The main motivation behind the development of this rehabilitation device was to realize a simple, cost-effective device for administering assisted upper-extremity (UE) therapy for post-stroke neurorehabilitation in both clinical and home environments. RUPERT III (the third version of the robot) consists of four degrees-of-freedom (DOFs) - each activated by a single "McKibben" type pneumatic muscle actuator (PMA) [10]. Each DOF of the robot is instrumented with a position sensor to measure joint angle, and a pressure sensor to sense the internal PMA pressure.

The primary feature of RUPERT that makes it unique among the existing UE exoskeleton robots is its wearability; every other UE robot has a grounded 


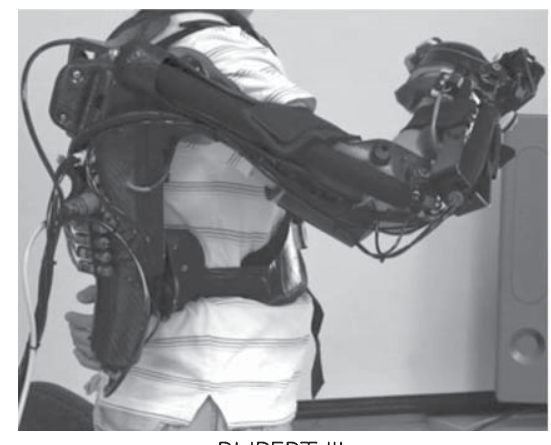

RUPERT III

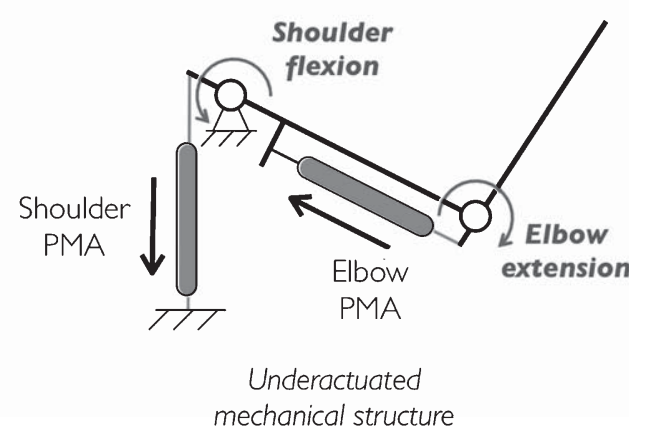

Fig. 1. Picture of RUPERT III (left panel) and a schematic representation of the underactuated design (showing only the shoulder and elbow joints) of the robot (right panel).

mechanical structure that is fixed to a static structure. The wearable nature of the robot allows it to be used for training therapy tasks in different positions mimicking real world scenarios (a seated or a standing position). However, this requires the robot's mechanical structure to be both compact and lightweight. These two crucial requirements were achieved through an under-actuated robotic design, which takes the form of unidirectional actuation in each DOF, resulting from the use of a single PMA to actuate the robot's individual DOFs (right panel of Fig. 1). Thus, each DOF of the robot can only be actuated in one direction; the actuation in the other direction is achieved either through the gravitational pull on the robot or the residual motor control a subject wearing the robot. The under-actuated design approach reduces the total number of actuators in the robot by half, which helps keep its mechanical structure simple, compact, lightweight, and also cost-effective.

The design of robotic assistance is one of the most important aspects of robot-assisted neurorehabilitation. The nature of the robotic assistance controls directly the level of voluntary participation from a subject during therapy, and indirectly their motivation. Providing a subject more than the necessary amount of assistance for a particular task can lead to "slacking" behaviour [11]. On the other hand, low levels of assistance will result in unsuccessful task performance, which can, over several movement trials, affect a subject's motivation through physical or mental fatigue. Thus, there have been several studies on the development of adaptive schemes for optimizing robotic assistance to "assist-as-needed" [11-14]. However, all these existing assistance schemes were developed and tested on robots with a grounded and fully actuated mechanical structure, unlike RUPERT III. Thus, it remains to be demonstrated that an adaptive robotic assistance scheme can be developed for an underactuated rehabilitation robot such as RUPERT to assist therapy tasks in a manner that encourages voluntary participation from a subject.

Previously, we had used a simple open loop controller for assisting stroke survivors to perform reaching tasks [9]. In the current article, we present the design and testing of two adaptive feedback controllers used for implementing two different robotic therapy modes for practicing reaching movements. The presented adaptive schemes modulate the amount of assistance depending on the motor ability of the stroke subject. The primary objective of the current study is to demonstrate that RUPERT with its simple underactuated mechanical design can be used for assisting therapy tasks in a manner that encourages voluntary participation from a subject.

The following sections of the paper describe the technical details of the adaptive feedback controllers, their performance evaluation on stroke survivors, and a critical discussion of the proposed controllers.

\section{Methods}

\subsection{System overview}

The schematic of the RUPERT system for administering upper-extremity rehabilitation therapy is shown in Fig. 2. RUPERT III is a wearable upperextremity exoskeleton robot with four DOFs - shoulder flexion/extension, elbow flexion/extension, elbow supination/pronation, and wrist flexion/extension. In the current study, the focus was on the conceptual 


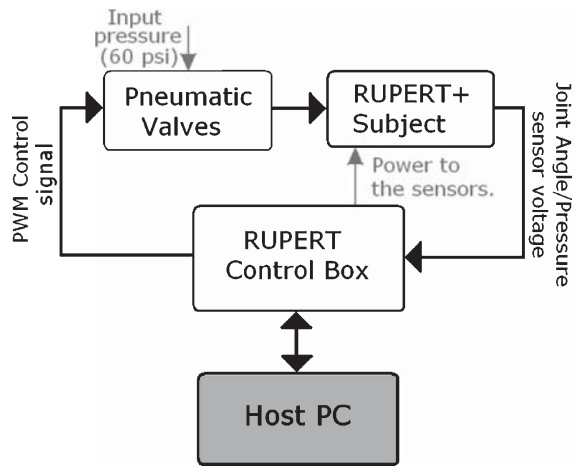

(a) RUPERT system overview

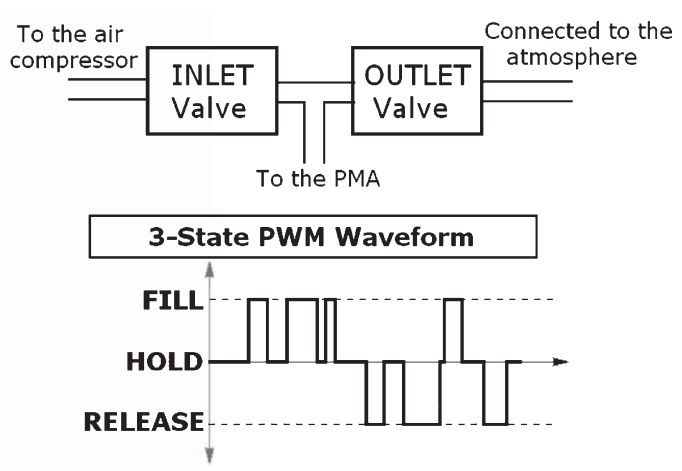

(b) 3-State PWM On/Off valve pair

Fig. 2. Schematics of the RUPERT system and the 3-state ON/OFF valve pair.

design and implementation of the adaptive control algorithms especially for reaching movements executed using the shoulder and elbow joints; this is one of the basic tasks that can be trained with RUPERT. Thus the distal DOFs - wrist flexion/extension and elbow supination pronation - are not discussed in the article. It should, however, be noted that the controllers presented in this study can be easily extended to the distal DOFs of the robot.

The 'RUPERT control box' (Fig. 2(a)) is a PC104 embedded computer (PCM 3370, Advantech, Inc.) with the associated data acquisition cards and other interface circuits. The 'RUPERT control box' interfaces to the robot to - (a) read the sensors in the robot; (b) control the air in the PMAs actuating the robot; and (c) communicate with the host computer. The 'Pneumatic Valves' (Fig. 2(a)) block consists of two on/off valves pairs (M10SA411M000060, Micro-Air series valve, Numatics Inc.), each controlling the bidirectional flow of air into a PMA. Each of these on/off valve pairs is a 3 -state valve that is controlled through a 3-state pulse width modulation (PWM) scheme (Fig. 2(b)). The host computer is a desktop PC that displays the biofeedback interface for training therapy tasks, and also acts as the interface for a clinician to use RUPERT for administering robot-assisted therapy.

\subsection{Robot controller overview}

The schematics of the robot controller developed for assisting reaching movements with RUPERT are shown in Fig. 3. The overall architecture of the controller consists of two feedback loops - the inner loop and the outer loop. The inner-loop performs control functions at the level of individual joints, while the outer-loop carries out higher-level functions such as decision-making and iterative learning. The controllers depicted in Fig. 3(a) and Fig. 3(b) was used to implement two robotic therapy modes: Adaptive active-assist mode (AAM) and Adaptive cooperative mode (ACM), respectively.

\subsubsection{Adaptive active-assist mode}

The active-assist mode is one of the most popular methods implemented in various existing UE rehabilitation robots for practicing robot-assisted movements $[5-7,15]$. The basic principle behind this therapy mode is to allow a subject to make a voluntary attempt to perform a given task. During this process, if the subject is unable to complete the task voluntarily, external assistance is provided for task completion.

The most essential design criterion for an activeassist therapy mode is to decide when to initiate robotic assistance, i.e. detecting when a subject is unable to perform the given task. All existing implementations of the active-assist therapy mode, in general, depend purely on the information obtained from a subject's instantaneous movement kinematics or kinetics to trigger robotic assistance. Some of the variables that are used in existing implementations are - spatial error, limb velocity, force generated by the subject, elapsed time, and even muscle activity as detected by surface EMG [16]. However, relying only on movement kinematics to trigger robotic assistance can lead to either premature or delayed initiation of assistance; both of these scenarios are undesirable. Premature initiation of robotic assistance can encourage "slacking behaviour" from the subject and thus reduce his/her voluntary 


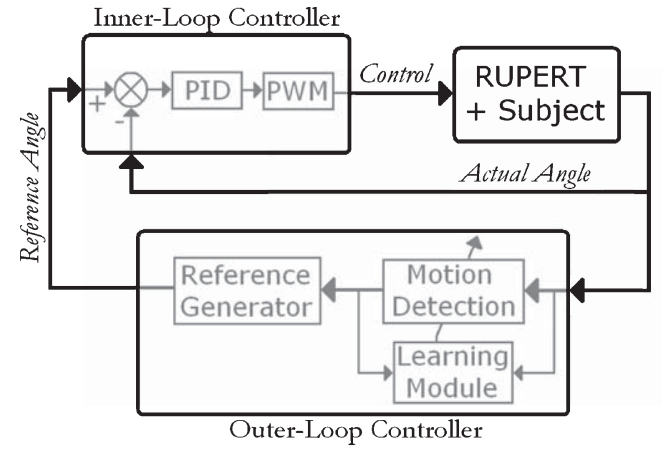

(a) Adaptive Active-Assist Mode (AAM)

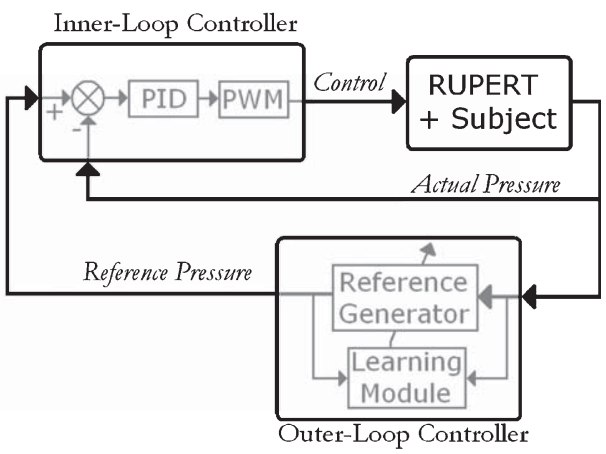

(a) Adaptive Co-operative Mode (ACM)

Fig. 3. Schematic of the RUPERT controllers used for the adaptive active-assist and adaptive co-operative modes.

participation. On the other hand, when there is delayed initiation of assistance, the subject might be voluntarily trying (at his/her maximum ability) to complete the given task without any success. In such a scenario, successive repetitions with long periods of unsuccessful voluntary attempt might lead to physical and mental fatigue that can be detrimental to the subject's motivation, and thus motor learning.

One of the possible ways to initiate robotic assistance at the appropriate moment, in an active-assist therapy mode, is to make use of information about a subject's motor ability, along with the real-time movement kinematics. One potential candidate for a measure of a subject's motor ability is the reachable workspace $\left(S_{R}\right)$. Reachable workspace - a kinematic measure of a subject's motor ability - is defined as the set of all points in space that can be reached by a subject voluntarily; space, in this definition, can be either the task space or the joint space of the arm. In the case of an impaired arm, $\left(S_{R}\right)$ is less than the total workspace $\left(S_{T}\right) ; S_{T}$ is equal to $S_{R}$ if the arm is unimpaired. The difference between $S_{R}$ and $S_{T}$ is defined as the unreachable workspace $\left(S_{U R}\right)$ of the arm, and the interface between $S_{R}$ and $S_{U R}$ is defined as the boundary of the reachable workspace $\left(B_{R}\right)$. A pictorial representation of the different terms described above is shown in Fig. 4(a) (some of terms in Fig. 4(b) are defined later in this section).

In order to understand how information about $S_{R}$ can be used to trigger robotic assistance in an active-assist therapy mode, consider an example of a reaching task to a target located in $S_{U R}$ (Fig. 4(a)). While performing the reaching movement, the subject will be able to move fairly well within his/her $S_{R}$. In this situation the initiation of robotic assistance must be avoided or delayed as much as possible, even when the subject's movement is slow or the subject, transiently, stops or moves away from the target; this reduces the chances of premature initiation of robotic assistance. On the other hand, once the subject gets close to $B_{R}$ or crosses into $S_{U R}$ (which can happen as a result of the trial-totrial variability in a subject's movement), the subject's movement will slow down and eventually come to a stop. Thus, in this situation, initiating robotic assistance immediately after the subject's movement slows down will prevent any unnecessary delay in providing assistance for task completion.

The detailed schematic of the controller developed for implementing the AAM is shown in Fig. 3(a). For the AAM, the inner-loop controller consists of two independent PID-like feedback joint position controllers, one each for the shoulder and elbow flexion/extension DOFs. The controllers are termed PID-like because of the modifications (listed in Table 1) introduced into a basic PID control structure.

The outer-loop controller houses three main subsystems: motion detection, reference generator, and learning module (Fig. 3(a)).

Motion Detection - This subsystem uses the realtime movement kinematics of a subject, along with the knowledge of his/her reachable workspace to trigger robotic assistance. For the real-time movement kinematics, the normalized endpoint velocity is used.

$$
V_{E P}^{N}(t) \triangleq \frac{V_{E P}(t)}{\max _{x \in[0, t]} V_{E P}(x)}
$$

where, $V_{E P}(t) \triangleq \frac{d}{d t}\left[r_{E P}(t)\right]$ is the endpoint velocity, and $r_{E P}(t)$ is the distance between the target location and the arm's current endpoint location. 


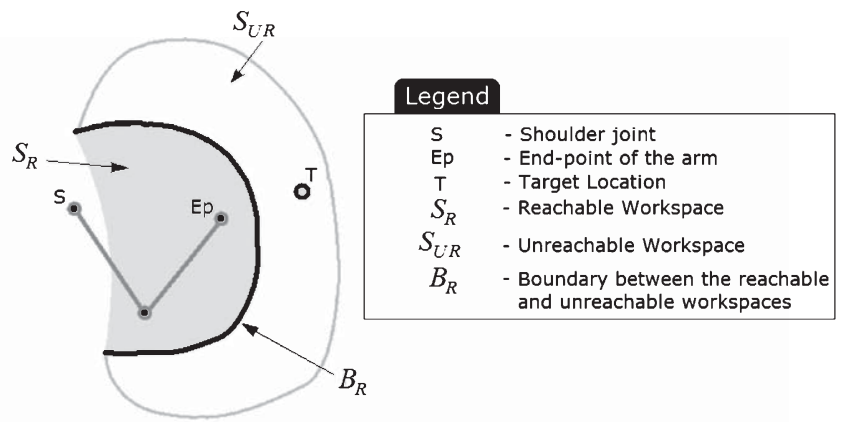

(a) Illustration of the idea of reachable and unreachable workspaces.

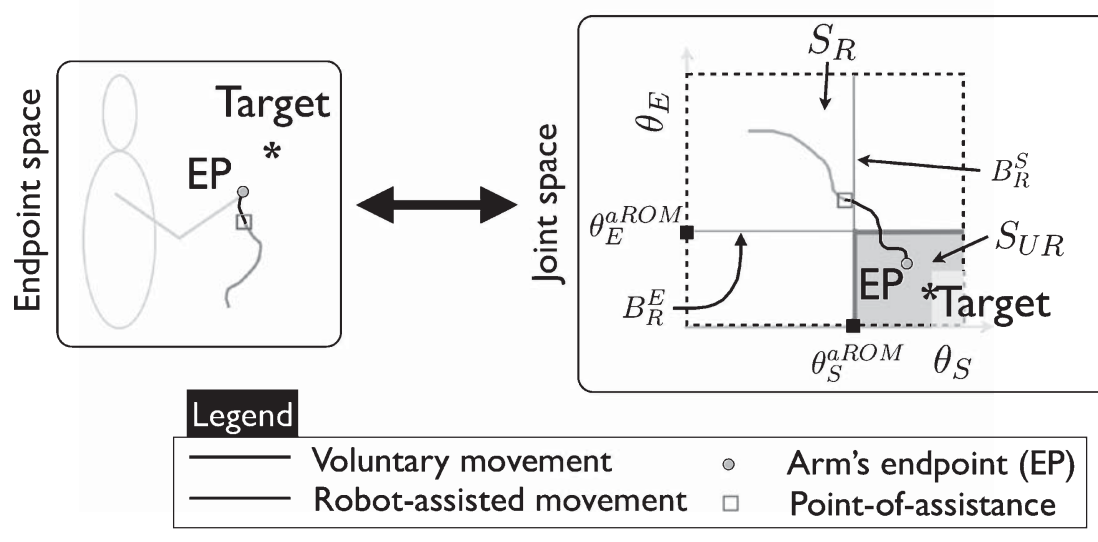

(b) Representation of reachable and unreachable workspaces (in the joint space) used in the AAM.

Fig. 4. Illustration of the idea of reachable workspace used in the adaptive active-assist therapy mode.

Table 1

Modifications to the PID structure used in the inner-loop controller

\begin{tabular}{ll}
\hline Modification & \multicolumn{1}{c}{ Rationale } \\
\hline $\begin{array}{l}\text { Limiting the output of the } \\
\text { integral term }\end{array}$ & To prevent integrator windup \\
$\begin{array}{c}\text { The output of the controller is } \\
\text { limited to within }+1 \text { and }-1\end{array}$ & $\begin{array}{c}\text { This is the range of signal } \\
\text { required for generating the } \\
\text { PWM signal }\end{array}$ \\
$\begin{array}{c}\text { The differential term of the } \\
\text { controller is implemented } \\
\text { using a high pass filter }\end{array}$ & $\begin{array}{l}\text { Thish fo prevent amplification of } \\
\text { high frequen noise, which } \\
\text { can make the control signal } \\
\text { very noisy, and thus generate a } \\
\text { lot of chatter in the pneumatic } \\
\text { valves }\end{array}$ \\
\hline
\end{tabular}

The reachable workspace of a subject is represented in the joint space in the AAM. The joint space, in this particular situation, is a two dimensional space spanned by the shoulder flexion/extension $\left(\theta_{S}\right)$ and the elbow flexion/extension $\left(\theta_{E}\right)$ joint angles. A mathematical description of $S_{R}$ can take many different forms. It depends on the level of details required in the representation of $S_{R}$, which is dictated by the application. In the current application, the simplest possible representation for $S_{R}$ was used.

$$
\begin{aligned}
S_{R} \triangleq & \left\{\left(\theta_{S}, \theta_{E}\right) \mid B_{R}^{S}\left(\theta_{S}\right)<0, B_{R}^{E}\left(\theta_{E}\right)<0\right\} \\
& B_{R}^{S}\left(\theta_{S}\right) \triangleq \theta_{S}-\theta_{S}^{a R O M} \\
& B_{R}^{E}\left(\theta_{S}\right) \triangleq \theta_{E}^{a R O M}-\theta_{E}
\end{aligned}
$$

where, $\theta_{S}^{a R O M}$ and $\theta_{E}^{a R O M}$ are the active range of motion of the shoulder flexion/extension and elbow flexion/extension DOFs, respectively. These two parameters, $\theta_{S}^{a R O M}$ and $\theta_{E}^{a R O M}$, physically correspond to the amount by which subjects can voluntary flex the shoulder and extend the elbow respectively.

The definition of $S_{R}$ given in Eq. 1 segments the joint space into a reachable and unreachable workspace (depicted in Fig. 4(b)). It should be noted that this particular definition is a crude approximation of the actual reachable workspace, and its use is motivated by the simplicity of its definition, which requires only two 
Table 2

Description of the rules used by the motion detection module in the AAM

\begin{tabular}{lc}
\hline Situation & Rule \\
\hline Situation-1: & Activate robotic assistance if the subject \\
$\left(\theta_{S}, \theta_{E}\right) \in S_{R}$ & has not moved towards the target \\
& $\left(V_{E P}^{N}(t) \geq 0\right)$, cumulatively, for at least \\
& 3 seconds \\
Situation-2: & Activate robotic assistance if the subject \\
$\left(\theta_{S}, \theta_{E}\right) \in S_{U R}$ & slows down $\left(V_{E P}^{N}(t) \leq 0.25\right)$ for the \\
& last 0.25 second \\
\hline
\end{tabular}

parameters $\left(\theta_{S}^{a R O M}\right.$ and $\left.\theta_{E}^{a R O M}\right)$ for its identification.

The kinematic variable $V_{E P}^{N}(t)$, and the estimate of a subject's reachable workspace are used in two specific rules to decide when to initiate robotic assistance (Table 2). These two rules correspond to two different situations, namely when the subject is within (situation-1) or outside (situation-2) the current estimate of his/her reachable workspace.

Reference Generator - This subsystem generates the reference signals for the inner-loop position controllers. Prior to the initiation of assistance, the movement made by the subject is purely voluntary. This is ensured by providing the inner-loop position controllers with a reference signal $\left(\theta_{x}^{r}(t)\right)$ that is equal to the actual joint position; thus effectively disabling them (the error seen by the position controllers is uniformly zero). However, once robotic assistance is initiated, the inner-loop position controllers are provided with the appropriate reference signals to complete the therapy task. The individual reference signals - based on the minimum jerk equation (MJT) [17] - smoothly connect the values of the joint angles, at the time when robotic assistance is initiated (this is referred to as point-of-assistance in the following sections), to the corresponding target joint angles (Fig. 5). A smooth interface between the actual

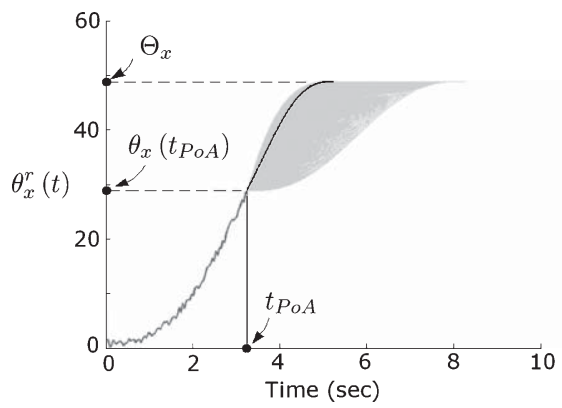

movement signal and the reference signal (shown in Fig. 5) is achieved through an optimization procedure. This procedure optimizes the parameters of the minimum jerk equation to get the best possible transition between the actual movement signal and the reference signal at the point-of-assistance $\left(t_{p o A}\right)$.

Learning Module - The purpose of the learning module is to estimate $S_{R}$. The 'Learning Module' iteratively estimates the parameters of $S_{R}$ (i.e. $\theta_{S}^{a R O M}$ and $\theta_{E}^{a R O M}$ ) from the previous movements. The iterative learning rule used to identify these parameters is as follows:

$$
\begin{aligned}
\theta_{x}^{a R O M}[j+1] \triangleq & \left(1-M_{j}\right) \times E\left(\theta_{x}^{j}\right)+M_{j} \\
& \times F\left(\Theta_{x}, \theta_{x}^{a R O M}[j]\right)
\end{aligned}
$$

where, $\theta_{x}^{a R O M}[j]$ is the estimate of the active range of motion for DOF $x$ for the $j$ th movement trial $\left(\theta_{x}^{a R O M}[1]=\Theta_{x}\right), \theta_{x}^{j}$ is the temporal record of the joint angle for the DOF $x$ for the $j$ th movement trial, $\Theta_{x}$ is the target angle for DOF $x, E(\cdot)$ is the estimating term, $F(\cdot)$ is the forgetting term, and $M_{j}$ is called the mode-of-assistance for the $j$ th movement trial. Modeof-assistance classifies a given active-assist movement into two categories based on the type of an active-assist movement.

- Type-1 - The subject completes the given task without any assistance.

- Type-2 - The subject completes the task with robotic assistance.

In the case of the AAM, Type-2 movements can be further classified into two categories:

- Type-2a - The subject crosses the current estimate of his/her reachable workspace, and is provided immediate assistance.

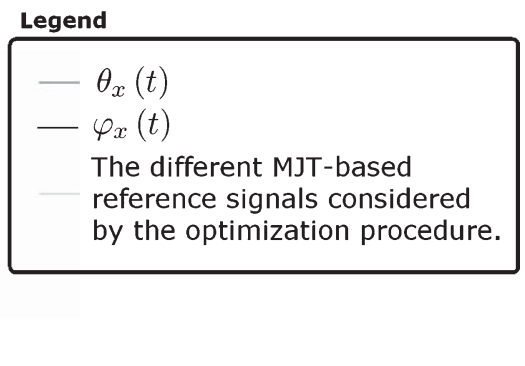

Fig. 5. Demonstration of the optimization procedure used for generating the reference signal for the inner-loop position controllers in AAM. 
- Type-2b - The subject does not cross the current estimate of his/her reachable workspace, and thus was provided delayed assistance.

$$
M_{j}=\left\{\begin{array}{l}
1, \text { for Type } 1 \text { and Type } 2 a \text { movements } \\
0, \text { for Type } 2 b \text { movements }
\end{array}\right.
$$

A reasonable estimate of the parameters $\left(\theta_{S}^{a R O M}\right.$ and $\theta_{E}^{a R O M}$ ), which can be obtained from the joint kinematics of an active-assist movement trial, is the value of the joint angles for the two DOFs when robotic assistance is initiated $\left(\theta_{x(p o A)}^{j}\right)$. The $E(\cdot)$ term calculates $\theta_{x}^{a R O M}$ by estimating the mean $\bar{\theta}_{x(p o A)}[j]$ and standard deviation $\sigma_{\theta_{x(P o a)}}[j]$ of $\theta_{x(p o A)}^{j}$ for the last few movement trials.

$$
\begin{gathered}
\bar{\theta}_{x(P o A)}[j]=\frac{1}{m} \sum_{i=\max (1, j-N+1)}^{j} \theta_{x(P o A)}^{i} ; \\
\mathrm{m}=\max (j-N+1, j) \\
\sigma_{\theta_{x(P o A)}}[j]=\sqrt{\frac{1}{\mathrm{~m}-1} \sum_{i=\max (1, j-n+1)}^{j} \times\left(\theta_{x(P o A)}^{i}-\bar{\theta}_{x(P o A)}[j]\right)^{2}}, j>1 \\
\sigma_{\theta_{x(P o A)}}[0]=0
\end{gathered}
$$

where, $\theta_{x(P o A)}^{j}=\theta_{x}^{j}\left(t_{P o A}[j]\right.$, is the value of the joint angle for the $x \mathrm{DOF}$ at the time corresponding to the point of assistance for the $j$ th movement trial $\left(t_{\mathrm{PoA}}[j]\right)$. The $j$ th movement trial is the most recently completed movement trial. $\bar{\theta}_{x(P o A)}[j]$ is the mean of $\theta_{x(P o A)}^{j}$, and $\sigma \theta_{x(P o A)}[j]$ is the standard deviation of $\theta_{x(P o A)}^{j}$ for the last $m$ movement trials. The mean and the standard deviation are used in the $E(\cdot)$ term to estimate the parameter $\theta_{x}^{a R O M}$ for the next movement trial.

$$
\begin{aligned}
E\left(\theta_{x}^{j}\right) & =\bar{\theta}_{x(P o A)}[j]+K_{x} \sigma \theta_{x(\text { Poa })}[j] \\
K_{x} & =\left\{\begin{array}{l}
-1, x=S \\
+1, x=E
\end{array}\right.
\end{aligned}
$$

From Eq. 4 it should be noted that $E(\cdot)$ is used only when the mode-of-assistance for the previous movement trial is 0 , i.e. the subject did not cross the reachable workspace. When a subject fails to cross the estimated boundary of the reachable workspace, the estimate is adjusted for the next movement trial based on previous movements.

On the other hand, the forgetting term $(F(\cdot))$ is used when the mode of assistance for the previous movement trial is 1 . The purpose of the forgetting term is to overestimate the subject's reachable workspace. This is achieved by letting the estimates of $\theta_{S}^{a R O M}$ and $\theta_{E}^{a R O M}$ move towards the corresponding target angles $\left(\Theta_{S}\right.$ and $\left.\Theta_{E}\right)$. The estimates of the two parameters are changed by the forgetting term in proportion to the difference between the current estimate of the parameter $\left(\theta_{x}^{a R O M}\right)$ and the corresponding target angle $\left(\Theta_{x}\right)$, as shown below.

$$
\begin{aligned}
F\left(\Theta_{x}, \theta_{x}^{a R O M}[j]\right)= & \theta_{x}^{a R O M}[j] \\
& +K_{x}^{F}\left(\Theta_{x}-\theta_{x}^{a R O M}[j]\right)
\end{aligned}
$$

where, $K_{x}^{F}$ is the forgetting rate.

\subsubsection{Adaptive co-operative mode}

The adaptive co-operative mode, unlike the activeassist mode, is based on the idea of enabling task completion by providing the appropriate level of mechanical support to a subject. The mechanical support provided by the robot helps compensate for the forces and torques (such as the weight of the arm, muscle tone, synergistic coupling between the different joints, etc.) that might prevent a subject from completing a therapy task. Under this mode, the subject and the robot work together to complete a given task. For any given movement trial, a fixed level of mechanical support is provided to the subject. This mechanical support has to be of the appropriate level to enable successful task completion; otherwise, the movement trial would be unsuccessful. Based on the success or failure of the current movement trial, the mechanical support from the robot is iteratively adjusted to enable the subject to successfully perform the therapy task, while at the same time encouraging voluntary participation from the subject. The schematic of the controller used for the ACM is shown in Fig. 3(b). The overall control scheme used for the ACM is similar to an iterative learning control scheme with a serial architecture [18]. The inner-loop controller of the ACM is a pressure controller. It consists of two independent PID-like feedback pressure controllers that control the pressure in the PMAs actuating the individual DOFs. The use of a pressure controller scheme can be thought of as an approximate form of force control. 
The purpose of the outer-loop controller in the ACM is to generate the reference signals for the inner-loop pressure controllers, and to modulate the parameters of these reference signals based on the subject's movement performance. The outer-loop controller for ACM consists of two subsystems: reference generator, and learning module.

Reference generator - This block generates the reference signal for the pressure controllers in the inner-loop. At the start of each movement trial, setting the reference signals to zero effectively disables the inner-loop pressure controllers. When the subject initiates movement, the outer-loop controller generates a non-zero reference signal based on the minimum jerk equation:

$$
\begin{gathered}
P_{x(\text { ref })}^{j}(t)=P_{x f}^{j} \times\left\{6\left(\frac{t}{T_{j}}\right)^{5}-15\left(\frac{t}{T_{j}}\right)^{4}\right. \\
\left.+10\left(\frac{t}{T_{j}}\right)^{3}\right\}
\end{gathered}
$$

where, $P_{x(r e f)}^{j}$ is the pressure reference signal for the DOF $x$ for the $j$ th movement trial, $t \in\left[0, T_{j}\right]$ is time, and $T_{j}$ is the time it takes the reference signal get to the final value of pressure $\left(P_{x f}^{j}\right)$ for the $j^{\text {th }}$ movement trial.

Learning Module - The 'learning module' modifies the parameter $P_{x f}^{j}$ of the reference signal (Eq. 5) based on a subject's performance. This parameter corresponds to the final pressure applied to the PMA actuating the DOF $x$; the higher the final pressure the greater the assistance for the corresponding DOF. The learning rule used to adjust the parameter $P_{x f}^{j}$ is the following:

$$
P_{x f}^{j+1}=\left(1-S_{j}\right) \times A\left(P_{x f}^{j}, \theta_{x}^{j}, \Theta_{x}\right)+S_{j} \times F\left(P_{x f}^{j}\right)
$$

$$
S_{j}=\left\{\begin{array}{l}
1, j^{\text {th }} \text { movement trial is successful } \\
0, j^{\text {th }} \text { movement trial is successful }
\end{array}\right.
$$

where, $A(\cdot)$ is the assistance term, $F(\cdot)$ is the forgetting term, and $S_{j}$ indicates the success or failure of the $j^{\text {th }}$ movement trial.

The assistance term $(A(\cdot))$ is used when a subject fails to complete a therapy task $\left(S_{j}=0\right)$. This term changes $P_{x f}^{j}$ in order to enable the subject to complete the given task successfully. The change in $P_{x f}^{j}$ is proportional to the current value of $P_{x f}^{j}$, and the constant of proportionality is a function of the difference between the target angle $\left(\Theta_{x}\right)$ and the value of the joint angle at the end of the $j^{\text {th }}$ movement trial

$$
A\left(P_{x f}^{j}, \theta_{x}^{j}, \Theta_{x}\right)=P_{x f}^{j}+K_{a}\left(\Theta_{x}-\theta_{x}^{j}\right) \times P_{x f}^{j} .(7)
$$

The forgetting term $(F(\cdot))$ is used when a subject successfully performs a given task $\left(S_{j}=1\right)$. The purpose of this term is to reduce the assistance provided by the robot, when the subject is able to successfully complete the therapy task. The forgetting term exponentially decays the current maximum reference pressure to zero with each successful movement trial

$$
F\left(P_{x f}^{j}\right)=P_{x f}^{j}-k_{f} \times P_{x f}^{j}
$$

where, $k_{f}$ is the forgetting rate.

\subsection{System testing}

The feedback controllers developed for RUPERT were tested on 3 stroke survivors. The testing procedure consisted of voluntary and robot-assisted reaching movements to different target locations. Reaching tasks were practiced in a simple non-virtual reality biofeedback environment (shown in Fig. 6). This biofeedback environment presents the sagittal view of arm reaching movements performed by a subject. It displays a simple two-link stick figure of the subject's arm and a circular target zone $\left(T_{\text {zone }}\right)$ that serves as the reaching target. This 2-link stick figure is driven by the joint angles sensed from the robot. The goal for the subject in these reaching movements is to move the endpoint of the stick figure into $T_{z o n e}$.

The subjects were recruited and consented to participate in the study, which consisted of a single testing session. The study was approved by the Institutional Review Board at Arizona State University. All subjects were screened by a certified physician/therapist to ensure that they satisfy the inclusion/exclusion criteria. The protocol used to test the two therapy modes is outlined in Table 3.

\section{Results}

The feedback controllers for implementing AAM and ACM were developed for RUPERT and tested on three stroke survivors. 


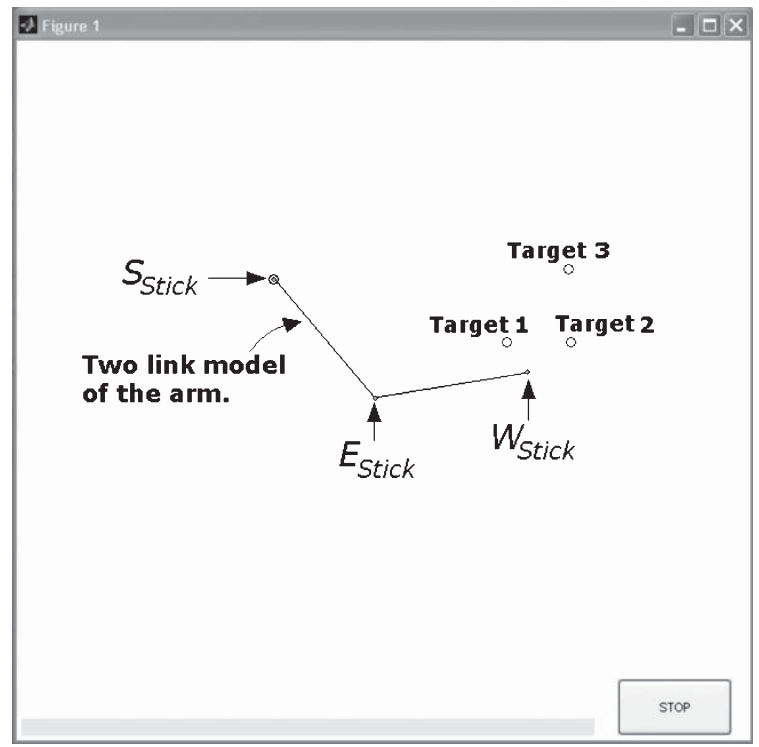

Fig. 6. Screenshot of the biofeedback environment used for practicing movements with the RUPERT system. $S_{\text {stick }}$ is the shoulder location, $E_{\text {stick }}$ is elbow location, and $W_{\text {stick }}$ is the location of endpoint.

Table 3

Description of the protocol used for testing the RUPERT controllers

\begin{tabular}{|c|c|}
\hline Step & Description \\
\hline $\begin{array}{l}\text { Step 1: Donn } \\
\text { RUPERT }\end{array}$ & Donn RUPERT on the subject \\
\hline Step 2: Set Targets & $\begin{array}{l}\text { Set the reaching targets for the subject. } \\
\text { Three targets are set in the arrangement } \\
\text { shown in Fig. } 6\end{array}$ \\
\hline $\begin{array}{l}\text { Step 3: Voluntary } \\
\text { Reaching }\end{array}$ & $\begin{array}{l}\text { The subject reaches to the set targets } \\
\text { voluntarily }\end{array}$ \\
\hline $\begin{array}{l}\text { Step 4: Passive } \\
\text { Reaching }\end{array}$ & $\begin{array}{l}\text { The robot moves the subject's arm to the set } \\
\text { targets. The subject is completely passive } \\
\text { in this mode. However, the subjects were } \\
\text { informed that the robot cannot perform } \\
\text { elbow flexion movements and thus, were } \\
\text { asked to perform the elbow flexion when } \\
\text { required to reach to the target }\end{array}$ \\
\hline $\begin{array}{l}\text { Step 5: ACM } \\
\text { Reaching }\end{array}$ & $\begin{array}{l}\text { The subject practices reaching movements to } \\
\text { each of the three targets with the robot } \\
\text { operating in the ACM }\end{array}$ \\
\hline $\begin{array}{l}\text { Step 6: AAM } \\
\text { Reaching }\end{array}$ & $\begin{array}{l}\text { The subject practices reaching movements to } \\
\text { each of the three targets with the robot } \\
\text { operating in the AAM }\end{array}$ \\
\hline
\end{tabular}

\subsection{Inner-loop position and pressure controllers}

The parameters for the position and pressure controllers (for the AAM and ACM, respectively) were obtained experimentally on healthy volunteers through trial-and-error basis. A fixed set of parameters was used for both the position and pressure controllers. In the case of the position controller, the application does not demand precise control of a subject's joint position, which was the primary reason for choosing a fixed parameter set. While for the pressure controller, a fixed parameter set was used because the load on the PMA does not drastically affect its pressure dynamics, and thus the performance of the pressure controller.

\subsection{Adaptive co-operative mode}

The results from the testing of the ACM are summarized in Figs. 7, 8. These two figures demonstrate the differential adaptation of the ACM to the motor abilities of the three different stroke subjects. Fig. 7 shows the plot of the endpoint trajectories of the three subjects performing reaching movements to Target- 2 . Each plot in this figure displays the following:

- The endpoint trajectory of the two-link stick model for both voluntary movements (grey trace) and ACM movements (black trace).

- The target zone in the form of a black circle indicating the intended reaching target (note that Fig. 7(c) has two concentric circles for the target zone, because two different target sizes were used for subject-3). 


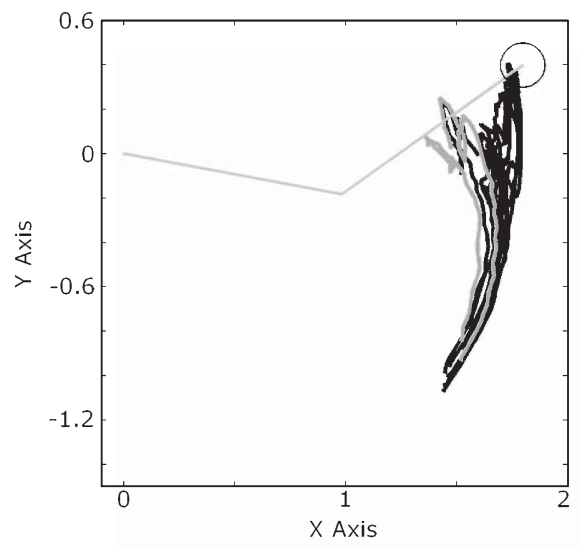

(a) Subject-I

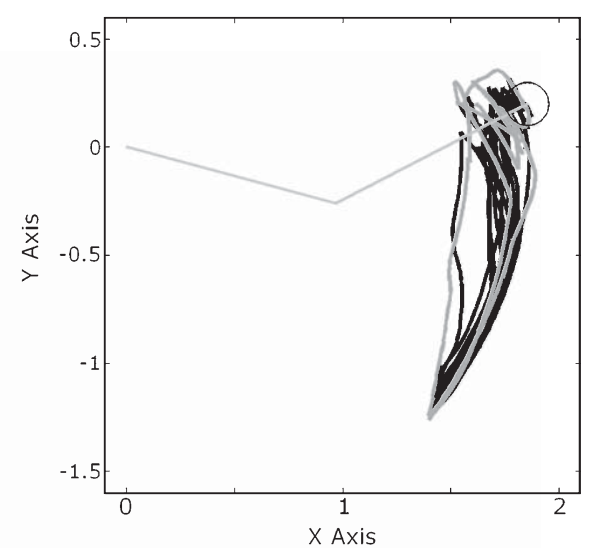

(b) Subject-2

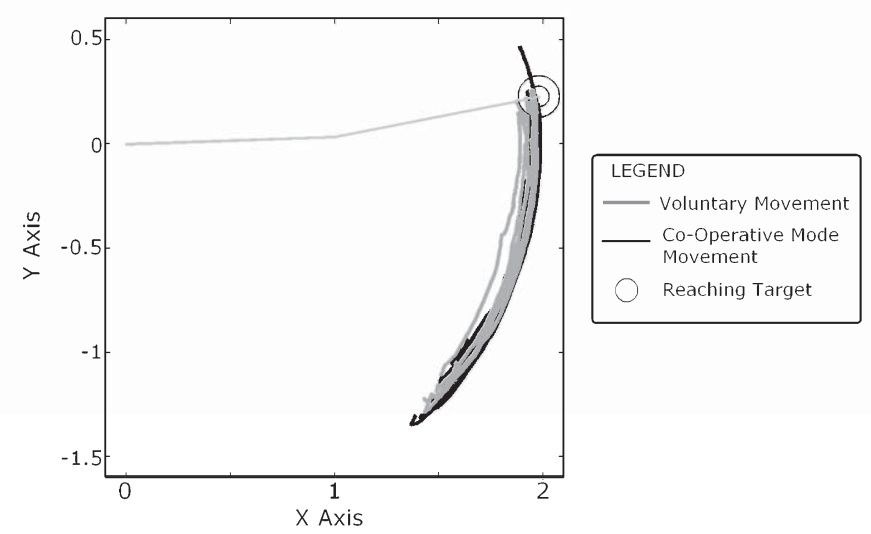

(c) Subject-3

Fig. 7. Endpoint trajectory for ACM reaching movements to Target-2 for the three stroke subjects.

- The desired final configuration of the two-link stick model (in lighter shade of grey) when its endpoint is at the centre of the target zone.

Figure 8 consists of six plots arranged in two rows and three columns. The three columns in this figure correspond to data from the three subjects. The first row displays the plot of $r_{\min (d i s t)}^{E P}$ as a function of the movement trial number; where $r_{\min (d i s t)}^{E P}$ is defined as the minimum distance between the arm's (two-link stick model) endpoint trajectory and the target location. This is a measure of how close the subject was able to get to the centre of the target zone. Each plot in the top row of Fig. 8 displays $r_{\min (d i s t)}^{E P}$ for both voluntary (grey trace) and ACM (black trace) movements. The dotted black lines in these plots represent the size of the target zone $\left(r_{\text {Target }}\right)$. A given movement trial is successful, if $r_{\min (d i s t)}^{E P}<r_{\text {Target }}$ for that particular movement trial, else the movement is unsuccessful.
The second row of plots in Fig. 8 displays the variables related to the pressure in the PMA controlling the shoulder flexion/extension DOF. Each of the three plots in the second row displays the following:

- $P_{S f}^{j}$ (Eq. 5) in a solid grey trace as a function of the movement trial $(j)$.

- $P_{S}^{M a x}[j]$ in a solid black trace as a function of the movement trial number $(j) . P_{S}^{M a x}[j]$ is the maximum value of the pressure in the PMA controlling the shoulder flexion/extension DOF for the $j$ th movement trial.

- The mean maximum pressure in the PMA controlling the shoulder flexion/extension DOF joint during passive movements; this is shown in a black dotted line.

The plots in Figs. 7, 8 demonstrate the ability of the ACM to adapt to the motor ability of different stroke 


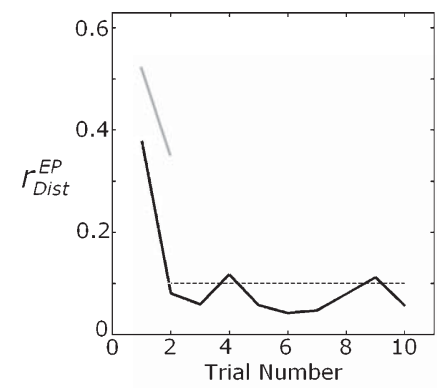

(a) Subject-I

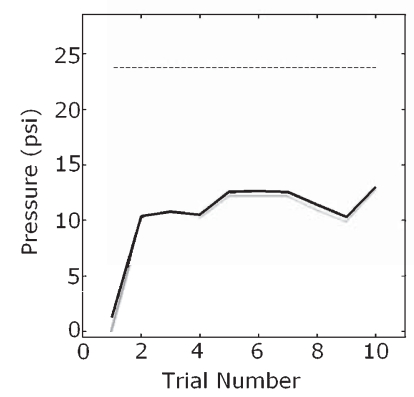

(a) Subject-I

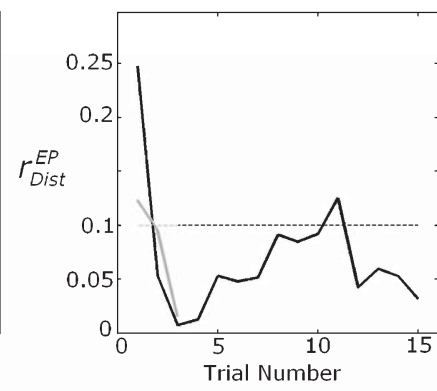

(b) Subject-2

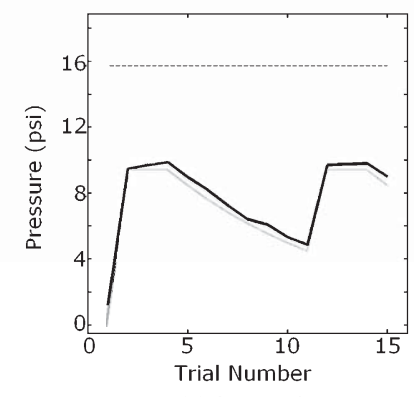

(b) Subject-2

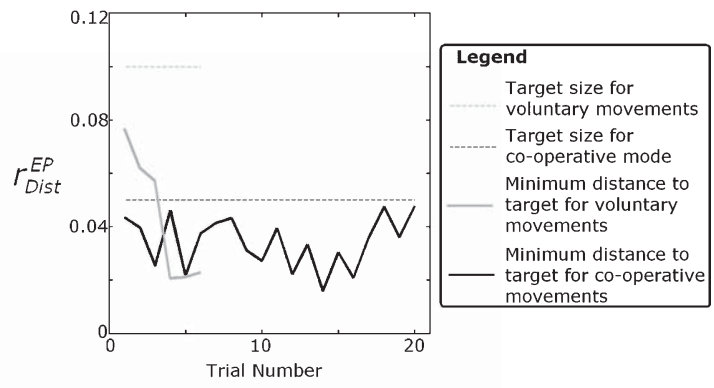

(c) Subject-3

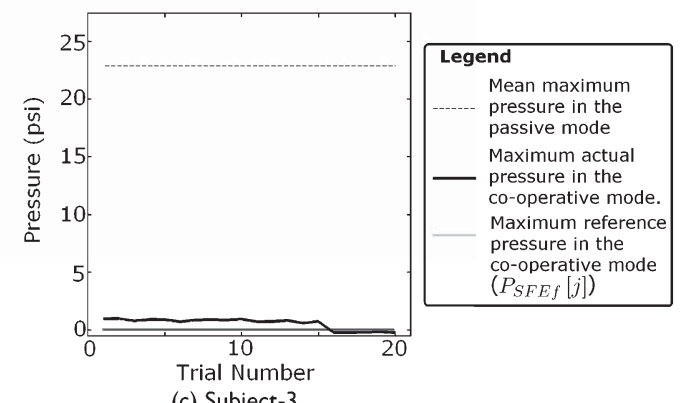

Fig. 8. Minimum distance to target for voluntary and ACM reaching movements to Target-2 (top row), and maximum shoulder PMA pressure for ACM reaching movements to Target-2 (bottom row) for all three stroke subjects.

subjects. Subject-3 was able to successfully reach Target-2 voluntarily, and also in the ACM (Fig. 7(c)), as demonstrated in Fig. 8(c) $\left(r_{\min (\text { dist })}^{E P}<r_{\text {Target }}\right.$ for all movements in both voluntary and ACM conditions). Subject-3 had a reasonable level of motor control in the affected arm, as evident not only from these successful reaching performances, but also from the consistency of the subject's endpoint trajectory (Fig. 7(c)). Therefore, no assistance (pressure) was provided to the subject by the robot in the ACM (the value of $P_{S f}^{j}$ is uniformly zero for all movement trials under the ACM (Fig. 8(c)).

In the case of subject-1 and subject-2, robotic assistance was provided in the ACM to enable successful task completion. From Figs. 7(a), 8(a) it can be seen that subject-1 was unable to reach the target voluntarily. Subject-2 (Figs. 7(b), 8(b)), on the other hand, was able to reach the target voluntarily only on some of the movement trials. However, both subject- 1 and subject2 were able to successfully reach the target with the appropriate level of assistance from the robot in the ACM. For both these subjects, the very first movement to the target under the ACM was unsuccessful, and thus a non-zero robotic assistance was provided for the subsequent reaching movements (Figs. 8(d), $8(\mathrm{e})$ ). After the first movement trial, the robotic assis- tance is increased, decreased or maintained at the same level for the following movement trials, depending on the success or failure of the preceeding reaching movements. A failed reaching movement increases the robotic assistance for the next movement trial, as seen in trials 1, 4 and 9 in Fig. 8(d) and trials 1 and 11 in Fig. 8(e). A successful reaching movement, however, either leaves the robotic assistance unchanged (trials 2, 3, 5 and 6 in Fig. 8(d), and trials 2, 3, 12 and 13 in Fig. 8(e)), or decreases the assistance in an exponential fashion for the next movement trial (trials 7 and 8 in Fig. 8(d), and trials 4 to 10 in Fig. 8(e)).

A similar type of adaptation was also observed for reaching tasks to the different target locations.

\subsection{Adaptive active-assist mode}

The results from the testing of the AAM are presented in two subsections to demonstrate the different features of this adaptive therapy mode and its associated learning scheme.

\subsubsection{Maximizing voluntary participation}

The main feature of the AAM is its ability to allow a subject to voluntarily attempt a therapy task close to his/her maximum ability before initiating robotic 


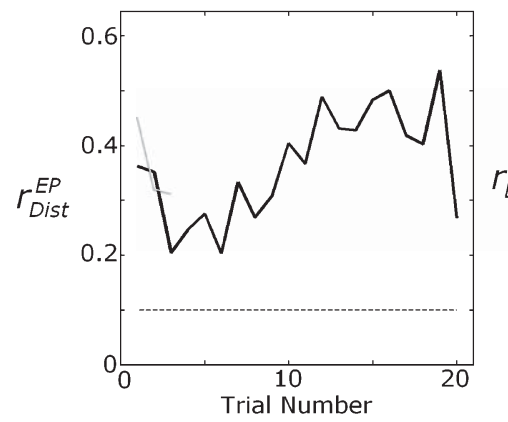

(a) Subject-I

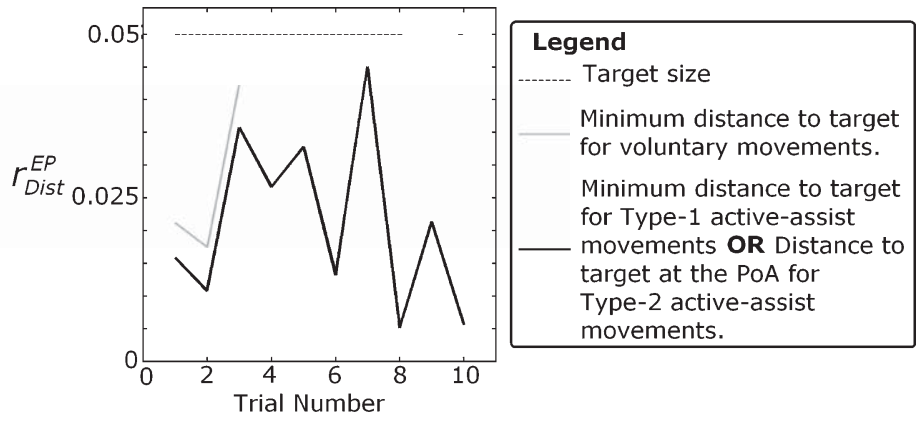

(b) Subject-3

Fig. 9. Comparison of the minimum distance to target for voluntary reaching movements and the distance from the target at which robotic assistance is initiated in AAM reaching movements for subject-1 and subject-3 reaching to Target -2 .

assistance for task completion. This feature of the AAM is demonstrated in Fig. 9. This figure consists of two plots that correspond to subject- 1 and subject- 3 performing AAM reaching movements. Each of these plots displays the following:

- $r_{\min (d i s t)}^{E P}$, the minimum distance to the target as a function of the movement trial number for voluntary movements (in solid grey trace); this is similar to the plots in the top row of Fig. 8.

- The minimum distance to the target for Type-1 AAM movements, and the distance to the target at $t_{P o A}$ for Type-2 AAM movements (in solid black trace).

- The size of the target zone in a dotted black line. It should be noted that the sections of the solid black trace that are below the dotted black line are Type1 AAM movements, and the rest are Type-2 AAM movements. For the grey trace, on the other hand, the sections of the trace that are below the dotted black line are successful reaching movements, while the rest are unsuccessful movements.

In Fig. 9(a), which corresponds to subject-1, all the 20 AAM movements shown in the figure are Type2 movements. It should be noted that in this figure the mean value of the black trace and that of the grey trace are approximately equal, which indicates that assistance was initiated, only after allowing the subject reach voluntarily as close as possible to the target. On the other hand, subject-3 (Fig. 9(b)) was able to complete all reaching movements without any assistance from the robot (all the movements are Type-1 AAM movements), since the subject had the ability to reach the target voluntarily (all points on the grey trace are below the dotted black line). These two plots, thus, demonstrate that the AAM does not initiate assistance when it is not required (subject-3), and initiates assistance only after allowing a subject reach to the maximum extent (subject-1).

\subsubsection{Learning the subject's motor ability}

The AAM uses an estimate of a subject's reachable workspace in order to allow for maximum voluntary participation from the subject when practicing reaching movements. The estimate is obtained on a trial-by-trial basis, iteratively, from the subject's previous movements. Fig. 10 depicts the workspace estimates for the three subjects reaching to their corresponding Target-3. The purpose of this figure is to demonstrate how the AAM learns the motor abilities of different subjects. Each of the three plots depicts the following information in the joint space:

- The target location, as indicated by an asterisk.

- The point on the joint space trajectory closest to the target for a voluntary movement (in black circles). The region occupied by black circles is represented by a lightly shaded grey colour rectangle. This rectangle is centred at the mean of these black circles and its dimension equals to twice the standard deviation of the scatter plot (formed by the black circles) along the corresponding axes. This region indicates how close a subject could get to the target voluntarily.

- The point in the joint space trajectory (shown in black squares) that is: (a) closest to the target for Type- 1 active assist movements or, (b) the point in the trajectory at the point-of-assistance for Type- 2 active-assist movements. The region occupied by 

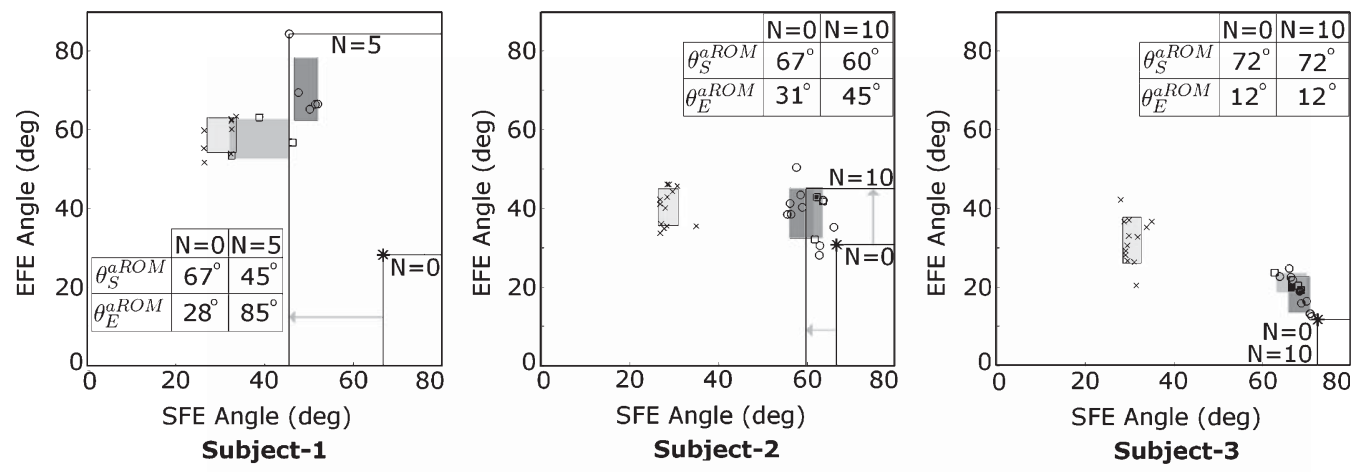

LEGEND

\begin{tabular}{|c|c|c|c|}
\hline \multirow{2}{*}{$\circ$} & \multirow{2}{*}{$\begin{array}{l}\text { Joint space location of the arm's endpoint } \\
\text { when it is closest to the target during } \\
\text { voluntary movements. }\end{array}$} & * & Target location in the joint space. \\
\hline & & \multirow[t]{2}{*}{$\longrightarrow$} & \multirow{2}{*}{ Boundry of the reachable workspace. } \\
\hline \multirow{3}{*}{$\square$} & \multirow{3}{*}{$\begin{array}{l}\text { Joint space location of the arm's endpoint } \\
\text { when it is closest to the target for Type-1 } \\
\text { active-assist movements OR at the PoA } \\
\text { for Type- } 2 \text { active-assist movements. }\end{array}$} & & \\
\hline & & $\square$ & Region occupied by ' 0 '. \\
\hline & & - & Region occupied by ' $₫$ '. \\
\hline$\times$ & $\begin{array}{l}\text { Joint space location of the initial position } \\
\text { of the arm's endpoint }\end{array}$ & $\square$ & Region occupied by ' $\times$ '. \\
\hline
\end{tabular}

Fig. 10. Demonstration of the learning of a subject's reachable workspace for AAM reaching movements to Target- 2 for the three stroke subjects.

these black squares is represented by a dark grey colour rectangle, similar to the one for voluntary movements. This region indicates how close to the target a subject was allowed to move before initiating assistance.

- The initial position of the joint space trajectory for each movement is shown in a black coloured ' $x$ '. The region occupied by these points is represented by light coloured rectangle with a black coloured edge. This region indicates the starting position of a subject when performing the reaching movements shown in the plot.

- The estimate of the reachable workspace for the first movement trial $N=1$ and a later movement trial $(N=5)$ for subject- 1 and $(N=10$ for subject- 2 and subject-3). It should be noted that the estimate for the two parameters $\theta_{S}^{a R O M}$ and $\theta_{E}^{a R O M}$ for the very first movement $(N=1)$ is always equal to the target angles.

- Each of the three plots in Fig. 10 also displays the value of the parameters $\theta_{S}^{a R O M}$ and $\theta_{E}^{a R O M}$ for the corresponding stroke subjects.

Figure 10 only shows the estimate of reachable workspace parameters at the start $(N=1)$ and at the end of a set of movement trials. It does not provide any information about the trial-to-trial dynamics of these parameter estimates. This is shown in Fig. 11(b), which corresponds to subject- 2 practicing reaching movements to Target- 3 in the AAM. This figure consists of three plots: Fig. 11(a) is similar to the plots shown in Fig. 9, and Fig. 11(b) shows the plot of $\theta_{S}^{a R O M}$ and $\theta_{E}^{a R O M}$ as a function of the movement trial number. Fig. 11(b) displays the following:

- The value of the parameters $\theta_{S}^{a R O M}$ and $\theta_{E}^{a R O M}$ in a thick black trace with each data point represented by a black circle.

- The target angle in a thin solid black line.

- The background of the movement trails indicates the type of the AAM movement trial. A Type-2b movement is depicted in a white background, while Type- $2 \mathrm{a}$ and Type-1 movements are depicted through a shaded background.

The estimate of the parameters for the reachable workspace is equal to the target angles for the first movement trial. For the consequent movement trials, the estimates are modified using the learning rule described in Eq. 4. For Type-2b movements, the parameters are updated based on their previous movement performance. While for Type-1 and Type-2a movements, the estimates are either not changed or moved towards the corresponding target angles. 

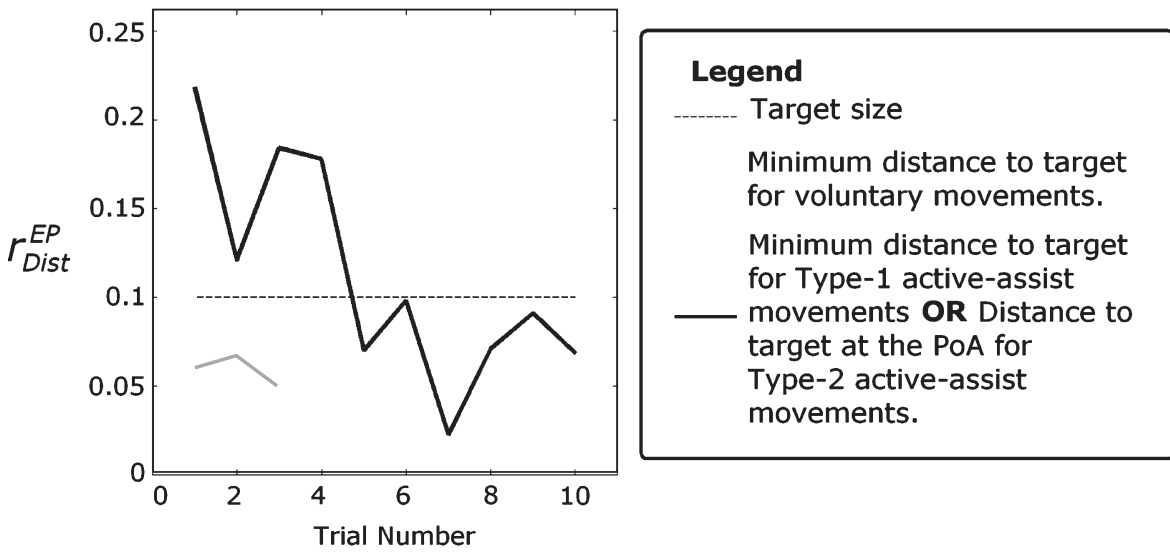

(a) Plot of the minimum distance to target as a function of movement trial number for voluntary and AAM movements for subject-2.
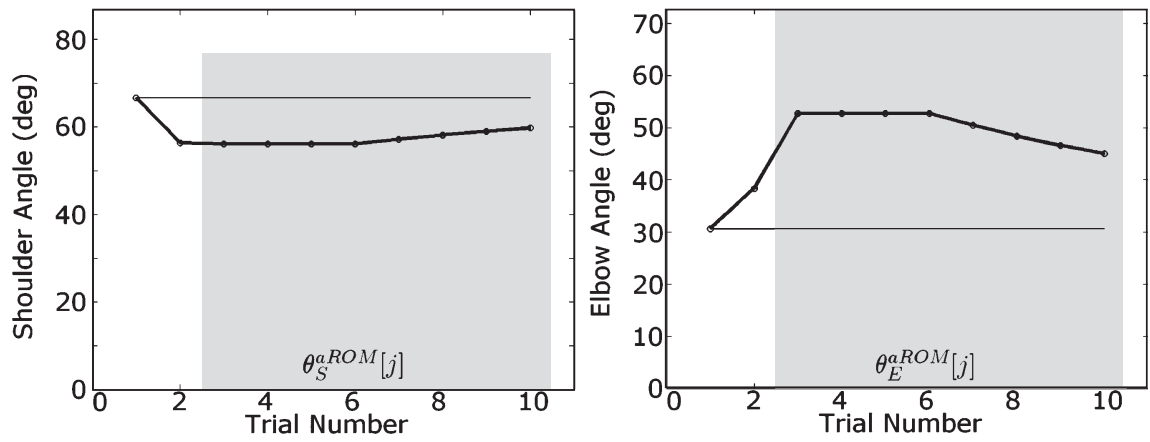

Legend

$\begin{array}{|ll|}- \text { Target Angle. } & \begin{array}{l}\text { Shaded background indicates that } \\ \text { the corresponding movement trial }\end{array} \\ \theta_{S}^{a R O M}[j] \text { or } \theta_{E}^{a R O M}[j] \text { as a } & \begin{array}{l}\text { corresponds to movement Type-1 } \\ \text { or Type-2a. Otherwise, it is a } \\ \text { function of the movement } j .\end{array} \\ \text { Type-2b movement. }\end{array}$

(b) Plot of estimated reachable workspace parameters for subject-2 as a function of movement trial number.

Fig. 11. Demonstration of the trail-by-trial learning of the parameters of reachable workspace for subject-2 reaching to Target-3.

\section{Discussion}

Feedback controllers for implementing two different adaptive robotic therapy modes were developed for RUPERT and were tested on three stroke subjects. The results from testing the two robotic therapy modes indicate that RUPERT, with its underactuated design, can be used for adaptive assisted UE task practice for movements such as reaching. The main feature of the two therapy modes presented in this study is their design to encourage voluntary participation from a stroke subject during robot-assisted task practice. A comparison of these two robotic therapy modes (AAM and the ACM) is provided in Table 4.

The AAM is a novel implementation of the activeassisted therapy practice idea. The uniqueness of the AAM is the use of information about a subject's motor ability, along with real-time movement kinematics, to initiate robotic assistance at the appropriate moment. Initiating robotic assistance at the appropriate moment reduces the chances of (a) 'slacking', when there is premature initiation of assistance, and (b) physical and mental fatigue, when the provision of assistance is delayed unnecessarily. This is demonstrated by the 
Table 4

Comparison of AAM and ACM

\begin{tabular}{|c|c|}
\hline Adaptive Active-Assist Mode & Adaptive Co-operative Mode \\
\hline $\begin{array}{l}\text { Complete a task for a subject } \\
\text { when he or she is not able } \\
\text { to do so voluntarily }\end{array}$ & $\begin{array}{l}\text { Enable task completion for a } \\
\text { subject when he or she is not } \\
\text { able to do so voluntarily }\end{array}$ \\
\hline $\begin{array}{l}\text { Implemented using a position } \\
\text { control scheme }\end{array}$ & $\begin{array}{l}\text { Implemented using a pressure } \\
\text { control scheme, which can } \\
\text { crudely be thought of as a force } \\
\text { control scheme }\end{array}$ \\
\hline $\begin{array}{l}\text { Any movement in this mode } \\
\text { can be separated into } \\
\text { voluntary robot-assisted } \\
\text { segments }\end{array}$ & $\begin{array}{l}\text { There is gradual blending of the } \\
\text { voluntary and robotic } \\
\text { contributions to the movement }\end{array}$ \\
\hline $\begin{array}{l}\text { Subjects successfully } \\
\text { complete each movement } \\
\text { trial, because of the use a } \\
\text { position control scheme }\end{array}$ & $\begin{array}{l}\text { Subjects are not successful on } \\
\text { each movement trial and } \\
\text { require a minimum level of } \\
\text { assistance to complete a task } \\
\text { successfully }\end{array}$ \\
\hline
\end{tabular}

results shown in Figs. 9, 10, which show that subjects were allowed to move as close as they can, voluntarily, to the target before initiating robotic assistance.

The information about a subject's motor ability, namely the reachable workspace, which is used in the AAM is estimated on a trial-by-trial basis. This feature of the AAM is demonstrated in Figs. 10, 11. An interesting pattern in Fig. 10 is the size of the reachable workspace for the three stroke subjects (indicated by the value of the parameters $\theta_{S}^{a R O M}$ and $\theta_{E}^{a R O M}$ shown in the individual plots). The size of the estimated reachable workspace for these stroke subjects correlates with the individual motor abilities of these three subjects. The estimated reachable workspace is smallest for subject-1, followed by subject- 2 , and subject-3 had the largest workspace of all three subjects. This information about a subject's reachable workspace is not only useful for triggering robotic assistance, but can also be used for tracking the changes in a subject's motor ability over the course of therapy. Since, the reachable workspace is estimated every movement trial, the trend in this variable can potentially help us understand the dynamics of the change in the subject's reaching ability induced by robot-assist therapy.

The use of a position control scheme in the AAM can be an issue of concern, because once robotic assistance is initiated, the robot might take complete control over subject's movement. However, it should also be noted that the underactuated design of the robot means the robot rarely takes complete control over a subject's motor ability. In the AAM, when robotic assistance is activated and the inner-loop position controllers are engaged, a subject will not experience any force from the robot when he/she moves faster than the robot. On the other hand, when the subject is unable to move (or move faster than the robot), the robot takes over and assists the subject, i.e. the robot takes control only when it is necessary. A similar idea was implemented in the MIT-MANUS robot through a modified impedance control algorithm that does not resist faster movements [13]. However, in the case of RUPERT this is an inherent feature that results of its basic mechanical design.

In the case of the ACM, it is not as straightforward as the AAM to ascertain the extent of voluntary contribution from a subject as both the subject and the robot work together, simultaneously, to achieve a given task. However, there are two important features in the results from the ACM movements shown in Fig. 8, which are noteworthy:

- For all the subjects, the maximum pressure required to reach the target in the ACM was lesser than the mean pressure required to move the subject's arm to the target in the passive mode. This indicates that the subjects were voluntarily contributing to the movement.

- In the plots for subject-1 and subject-2, there is a negative correlation between the maximum pressure and the minimum distance to the target. The minimum distance to target increases when the maximum pressure in the shoulder flexion/extension PMA is decreased (trials 7 to 9 for subject-1, and trials 4 to 11 for subject-2). This trend strongly suggests that subjects were making use of the assistance provided by the robot to complete the reaching. The fact that reducing assistance (maximum pressure) reduces movement performance (minimum distance to the target) is an indication that the provided assistance is close to the minimum required level for task completion for the given level of voluntary contribution from the subject.

One of the possible disadvantages of the ACM is that subjects don't always successfully complete a given therapy task, and thus might not experience the full afferent feedback associated with the given task. However, practicing therapy tasks with the ACM might be more motivating for a subject because, the ACM does not guarantee successful task performance on each movement trial, which is more challenging and game-like. 
A unique feature of the learning scheme used for implementing the AAM and ACM is that the learning scheme modifies the parameters of the controller, purely, based on the success or failure of the given therapy task, instead of using any specific movement kinematic measure. This means that there are no restrictions on the subject having to follow any specific spatial (a straight endpoint trajectory) or temporal (a smooth bell shaped velocity profile like the minimum jerk trajectory) movement pattern while performing a reaching task. This particular design was motivated by the following reasons:

- It results in a simple therapy mode that removes the need for choosing a specific movement kinematic pattern for the stroke subjects to follow. Additionally, there is no evidence in the existing literature that indicates the superiority of any particular movement pattern in improving motor performance of stroke subject.

- It might be unreasonable to expect a stroke subject with motor impairments to follow movement kinematic patterns that are observed in healthy subjects, such as a minimum jerk trajectory with a straight movement path for point-to-point reaching movements

- This gives more importance to function (reaching ability) rather than any specific movement kinematic variable. Improving function is likely to be more meaningful to the stroke subject rather than improving the kinematics of their movement.

- The underactuated design of RUPERT restricts the robot from enforcing any specific movement kinematics.

Additionally, it should also be noted that the adaptive therapy modes presented in this study are not specific to RUPERT, and can be implemented in other UE rehabilitation robots. The AAM mode is straightforward to implement in some of the existing UE robots - the current methods used for activating robotic assistance could be replaced by a scheme that makes of a measure the subject's motor ability. On the other hand, the ACM can be implemented as an adaptive stiffness control scheme that changes the robot's stiffness on a trial-by-trial basis based on the subject's movement performance (e.g. a high stiffness would correspond to a higher level of assistance). Moreover, combining ideas from the AAM and ACM are also possible. The use of the idea of a reachable workspace (from AAM) along with a stiffness control scheme (from ACM) can potentially result in an assistance scheme that encourages more voluntary contribution from a subject, than either AAM or ACM individually.

\section{Conclusion \& future work}

Two feedback control schemes for assisting reaching movements with RUPERT were developed and successfully tested on three stroke subjects. This study demonstrates that an underactuated wearable exoskeleton, such as RUPERT, can be used for administering robot-assisted therapy, in a manner that encourages voluntary participation from the subject undergoing therapy. Additionally, a novel implementation of an active-assist therapy mode for initiating robotic assistance at the appropriate moment was also presented.

Following the work presented in the current study, the next immediate step will be the optimization of the different parameters of the controllers developed for RUPERT to assist reaching movements. These controller parameters affect the amount of assistance provided to the subject while practicing robot-assisted movement, which directly affect the voluntary participation of the subject. This can be accomplished through small case studies that can help optimize the controller parameters, and also provide some preliminary evaluation of the usefulness of adaptive RUPERT-assisted therapy. These small case studies can then be followed by a controlled investigation of the therapeutic efficacy of RUPERT for training simple reaching movements in stroke subjects. Based on the existing literature on the use of robotic devices for UE stroke rehabilitation, we hypothesize that a therapeutic intervention administered using RUPERT, operating under either one of the two adaptive therapy modes developed in the current study, will be able to reduce motor impairments and improve the reaching ability of stroke subjects.

\section{References}

[1] S. Balasubramanian, J. Klein and E. Burdet, Robot-assisted rehabilitation of hand function, Curr Opin Neurol 23(6) (2010), 661-670

[2] B.R. Brewer, S.K. McDowell and L.C. Worthen-Chaudhari, Poststroke upper extremity rehabilitation: a review of robotic systems and clinical results, Top Stroke Rehabil 14(6) (2007), p. 22-44.

[3] D.J. Reinkensmeyer, et al., Emerging Technologies for Improving Access to Movement Therapy following Neurologic Injury, in Emerging and Accessible Telecommunications, 
Information and Healthcare Technologies-Emerging Challenges in Enabling Universal Access, Anonymous, Editor. (2002), IEEE Press.

[4] R. Riener, T. Nef and G. Colombo, Robot-aided neurorehabilitation of the upper extremities, Med Biol Eng Comput 43(1) (2005), p. 2-10.

[5] H.I. Krebs, et al., Robot-aided neurorehabilitation, IEEE Transactions on Rehabilitation Engineering 6(1) (1998), 75-87.

[6] D.J. Reinkensmeyer, et al., Design of robot assistance for arm movement therapy following stroke, Advanced Robotics 14(7) (2000), p. 625-637.

[7] C.D. Takahashi, et al., Robot-based hand motor therapy after stroke, Brain 131(Pt 2) (2008), 425-437.

[8] J.L. Patton and F.A. Mussa-Ivaldi, Robot-assisted adaptive training: custom force fields for teaching movement patterns, IEEE Trans Biomed Eng 51(4) (2004), p. 636-646

[9] T.G. Sugar, et al., Design and Control of RUPERT: A device for Robotic upper extremity repetitive therapy, IEEE Transactions on Neural Systems and Rehabilitation Engineering, 15(3) (2007), p. 336-346.

[10] F. Daerden and D. Lefeber, Pneumatic artificial muscles: actuators for robotics and automation, European journal of Mechanical and Environmental Engineering 47 (2000), p. 10-21.

[11] E.T. Wolbrecht, et al., Optimizing Compliant, Model-Based Robotic Assistance to Promote Neurorehabilitation, IEEE
Transactions on Neural Systems and Rehabilitation Engineering 16(3) (2008), p. 286-297.

[12] L.E. Kahn, W.Z. Rymer and D.J. Reinkensmeyer, Adaptive assistance for guided force training in chronic stroke, Conf Proc IEEE Eng Med Biol Soc 4 (2004), p. 2722-2725.

[13] H.I. Krebs, et al., Rehabilitation Robotics: Performance-based progressive Robot-assisted therapy, Autonomous Robots, 15(1) (2003), 7-20.

[14] L. Masia, et al., Performance adaptive training control strategy for recovering wrist movements in stroke patients: a preliminary, feasibility study, J Neuroeng Rehabil 6 (2009), p. 44.

[15] L.E. Kahn, et al., Robot-assisted reaching exercise promotes arm movement recovery in chronic hemiparetic stroke: a randomized controlled pilot study, J Neuroeng Rehabil 3 (2006), p. 12.

[16] L. Marchal-Crespo and D.J. Reinkensmeyer, Review of control strategies for robotic movement training after neurologic injury, J Neuroeng Rehabil 6 (2009), p. 20.

[17] T. Flash and N. Hogan, The coordination of arm movements: an experimentally confirmed mathematical model, The Journal of Neuroscience: the official journal of the Society for Neuroscience 5(7) (1985), 1688-1703.

[18] D.A. Bristow, M. Tharayil and A.G. Alleyne, A survey of iterative learning control. Control Systems Magazine, IEEE 26(3) (2006), p. 96-114. 

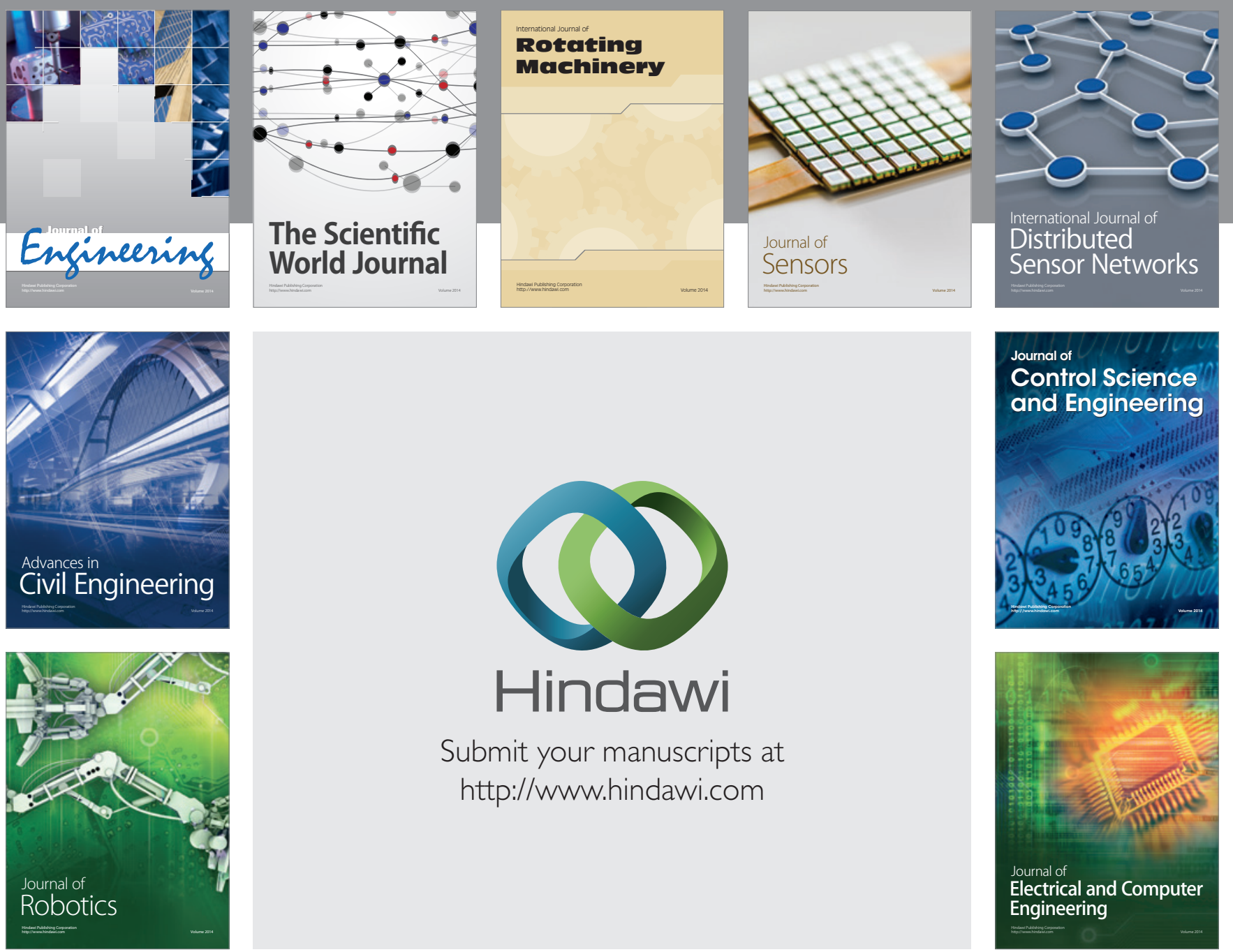

Submit your manuscripts at

http://www.hindawi.com
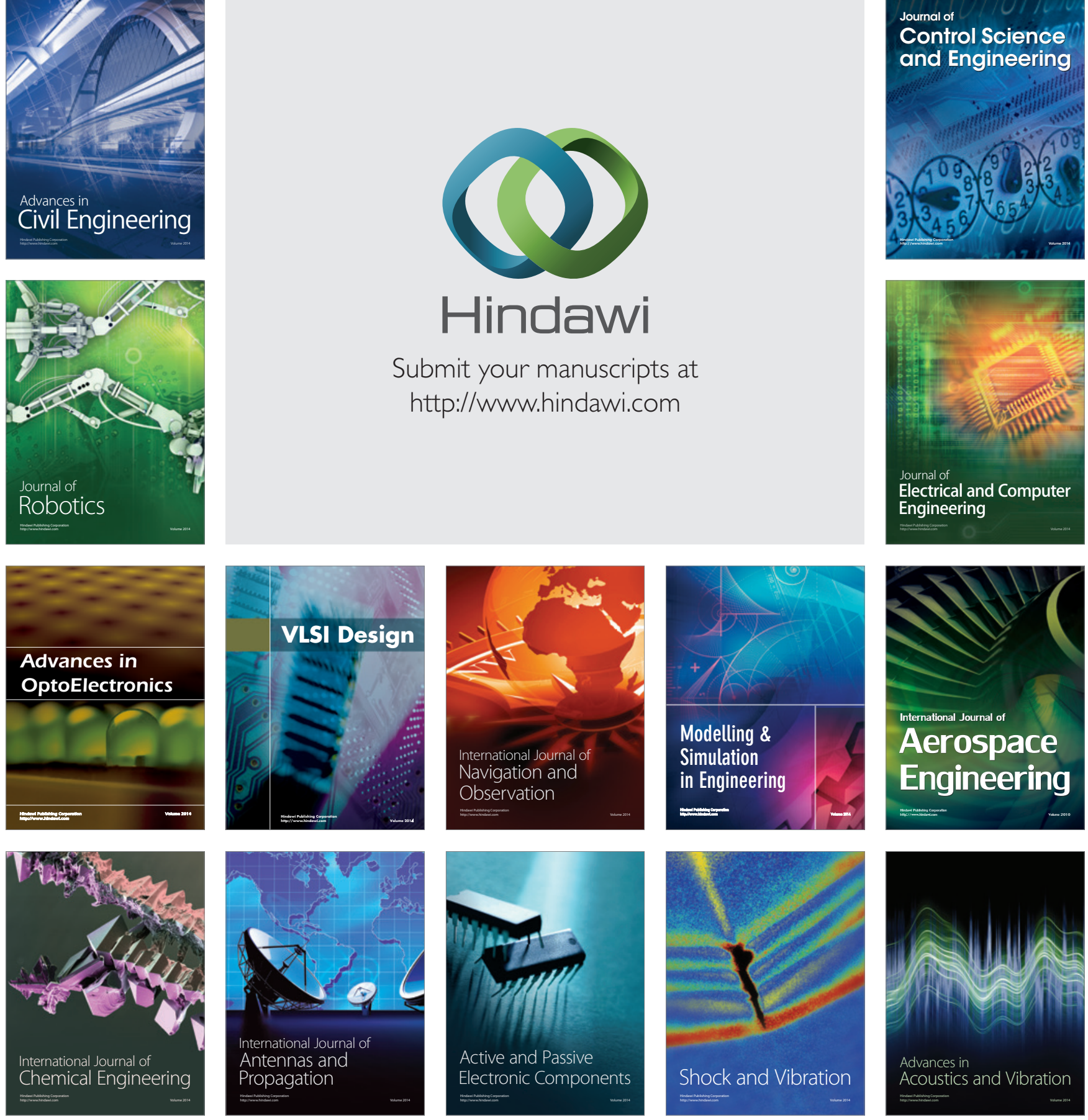